\title{
ARE GALAXIES OPTICALLY THIN TO THEIR OWN LYMAN CONTINUUM RADIATION? II. NGC 6822
}

\author{
Kanan Patel and Christine D. Wilson \\ Department of Physics and Astronomy, \\ McMaster University, \\ Hamilton, Ontario L8S 4M1 Canada
}

\begin{abstract}
In this paper we study OB stars, H II regions, and the state of ionization balance in the Local Group galaxy, NGC 6822. Using $H \alpha$ data and BV photometry of the blue stars in this dIrr galaxy, we investigate the distribution of OB stars and H II regions and determine whether individual areas of the galaxy are separately and/or collectively in a state of ionization balance. Four distinct components of the $H \alpha$ emission (bright, halo, diffuse and field) differentiated by their surface brightnesses are identified. We find that approximately $1 / 2$ of all OB stars in NGC 6822 are located in the field while only $1 / 4$ are found in the combined bright and halo regions, suggesting that OB stars spend roughly $3 / 4$ of their lifetimes outside "classical" H II regions. If OB stars escape from bright $\mathrm{H}$ II regions by destroying their parent molecular clouds, then cloud lifetimes after forming OB stars could be as low as $\sim 1-3 \times 10^{6}$ yrs or $1 / 4$ the typical main sequence lifetimes of OB stars. However, if the stars are simply escaping from the clouds without destroying them, then these data place no limits on molecular cloud lifetimes. We find that the entire field population of OB stars cannot have originated in and percolated out of existing $\mathrm{H}$ II regions. Comparing the observed $H \alpha$ emission with that predicted from stellar ionizing flux models and hydrogen recombination theory, we find that although the bright, halo and diffuse regions are probably in ionization balance, the field region is producing at least 6 times as much ionizing flux as is observed. The ionization balance results in NGC 6822 suggest that star formation rates obtained from $H \alpha$ luminosities must underestimate the true star formation rate in this galaxy by about $50 \%$. Comparing our results for NGC 6822 with previous results for the Local Group spiral galaxy M33, we find that the inner kiloparsec of M33 is in a more serious state of ionization imbalance, perhaps due to its higher surface density of blue stars. Thus the morphological class of a galaxy may be an important factor in how accurately we can determine star formation rates from $H \alpha$ luminosities.
\end{abstract}




\section{INTRODUCTION}

Evidence that massive OB stars are not always associated with optically luminous H II regions has been steadily increasing. Previous indications of this phenomenon included a study of the OB associations of M33 that showed many of the associations do not contain bright H II regions (Wilson 1990). As well, a study of the edge-on spiral galaxy, NGC 891, revealed the existence of filamentary $H \alpha$ structures high above the galactic disk which are not associated with any obvious ionizing sources (Rand et al. 1990). In addition, the neighbouring Large and Small Magellanic Clouds were found to house an entire population of OB stars that were located in the "field" far from any OB associations (Massey et al. 1994). These findings raise a number of interesting question, such as how are the OB star and H II region distributions related?

What fraction of the OB star lifetime is spent within an optically bright H II region? How prevalent are the "field" stars? What is the state of ionization balance in individual and collective regions of the galaxies?

The answers to these questions have important implications to issues in star formation and the interstellar medium, including the determination of extragalactic star formation rates (SFRs) and molecular cloud lifetimes. To answer these questions, we have investigated the OB and H II region population and the state of ionization balance within Local Group galaxies. We began with a study of the spiral galaxy, M33 (Patel \& Wilson 1995, hereafter Paper I). The present work is focused on the dIrr member of the Local Group, NGC 6822.

We begin by outlining some of the key issues (further details can be found in Paper I). The length of time an $\mathrm{O}$ star spends in the $\mathrm{H}$ II region phase, and thus partially embedded in its parent cloud, will have some bearing on the lifetime of the parent molecular cloud. Short lifetimes ( $10^{7}$ yrs Blitz \& Shu 1980) assume the embedding time is a large fraction of the total lifetime of the star so that the first $\mathrm{O}$ stars that form will disrupt their parent cloud. Molecular clouds undergoing periods of intense star formation followed by more quiescent star formation would result in long molecular cloud lifetimes $\left(\geq 10^{7} \mathrm{yrs}\right)$ so that each cloud could produce several OB associations of different ages (Elmegreen 1991). If O stars destroy their natal environments, then the fraction of $\mathrm{O}$ stars seen in the field or outside of bright $\mathrm{H}$ II regions (which is proportional to the length of time a typical O star spends outside a bright H II region) determines the time required to destroy the cloud. However, if these field O stars have simply moved out of their parent clouds, then cloud lifetimes could be considerably longer.

The second issue in this study concerns one of the most common methods of determining SFRs in extragalactic systems, namely using the $H \alpha$ luminosity. This method relies heavily on the assumption that the region in question is ionization bounded (Kennicutt 1983) i.e. all Lyman continuum photons produced by massive young stars are absorbed by the gas in the region. (The alternative scenario is for a region (or galaxy) to be density bounded, i.e. the Lyman photons are more than sufficient to ionize all the (dense) gas.) Since the observed $H \alpha$ luminosity is directly proportional to the Lyman continuum luminosity, by assuming an initial mass function (IMF) and theoretical models for the Lyman continuum luminosity as a function of stellar mass the "observed" Lyman continuum luminosity can be converted into a SFR for massive stars. The total SFR of a region is obtained by extending the IMF over the full range of stellar masses. In all of this, the assumption that the region is ionization bounded is crucial: since the SFR is directly proportional to the Lyman continuum luminosity and therefore the observed $H \alpha$ luminosity, a region out of which continuum photons are escaping will have a smaller estimated SFR than one that is ionization bounded. The assumption of ionization balance is not certain to be valid if a large fraction of the OB population lies outside bright H II regions. In view of the wide spread use of this technique in extragalactic systems, it is vital to verify this important assumption of the theory. 
Until very recently, ionization balance calculations in extragalactic systems had concentrated on individual OB associations in the Large and Small Magellanic Clouds (respectively, LMC and SMC). NGC 2122 and LH 118 in the LMC were found to be density rather than ionization bounded (Massey et al. 1989a) while NGC 346 in the SMC is, most likely, ionization bounded (Massey et al. 1989b). The results of the M33 study (Paper I) showed that the entire (inner $1 \mathrm{kpc}$ ) surveyed area, as well as individual ionized gas regions of the galaxy are not in ionization balance and in fact, in total, the surveyed region was producing between 3 and 7 times the observed flux.

At a distance of $0.5 \mathrm{Mpc}$ (McAlary et al. 1983), NGC 6822 is very well suited for our investigation. While its low galactic latitude makes this a somewhat difficult system to study, there is a substantial body of research in the Local Group literature that is associated with the galaxy. Photometric surveys of the stellar populations and OB associations include the photographic surveys of Hodge (1977), and most recently, CCD imaging in the B and V filters by Wilson (1992a). Luminous blue stars in NGC 6822 have been studied by Humphreys (1980), while the Wolf-Rayet star population has been surveyed by Armandroff \& Massey $(1991,1985)$. Over 140 H II regions of the galaxy have been catalogued and studied by Hodge et al. $(1989,1988)$. The recent evolutionary history, including determinations of the SFR within NGC 6822 have been discussed by Hodge et al. (1991) and Hodge (1980). Finally, the molecular gas content of the galaxy has recently received some attention with the CO surveys by Wilson (1992b), Ohta et al. (1993) and Wilson (1994).

In this paper, we use $H \alpha$ and BV photometric data of NGC 6822 to study the distribution of the $\mathrm{H}$ II regions and luminous blue stars (hereafter OB stars) in order to determine the fraction of an OB star's lifetime that is spent outside a bright H II region and hence, to address the issues of molecular cloud lifetimes after the formation of OB stars. With the aid of stellar ionization models, we use the data to determine whether the individual regions are separately and/or collectively in a state of ionization balance. Additionally, optical spectra of a number of stars in NGC 6822 have been used to test the performance of the principal ionization model used in the investigation. The implications of the state of ionization balance for the SFRs estimated from $H \alpha$ luminosities are also discussed. Finally we compare our results for NGC 6822 with the results obtained previously for M33. The analysis of the data ( $H \alpha$ and BV photometry),

discussion of the theoretical ionization models used and the limitation of these models appear in section $\S 2$. The OB star and H II region distributions are discussed in $\S 3$ and the findings of the ionization balance calculations appear in $\S 4$. M33 and NGC 6822 are compared in $\S 5$ and the results of our investigation are summarized in $\S 6$.

\section{SELECTION AND ANALYSIS OF DATA}

\subsection{PHOTOMETRIC DATA}

We use BV photometric data from Wilson (1992a) which had previously been used to study the OB associations in the galaxy. The observations were made at the Palomar 60 -inch telescope using the blue sensitive Tektronix chip, CCD 6 (chip scale $0.235^{\prime \prime}$ pixel $^{-1}$ ). The "T" shaped area surveyed included the main body of the galaxy as well as the northern bar region which contains many OB associations previously identified by Hodge (1977). The average seeing in B and V was, respectively, 1.4 arcsec and 1.3 arcsec. Additional details of the observations can be found in Wilson (1992a). The average photometric 
uncertainties are $0.03 \mathrm{mag}$ for $\mathrm{B}, \mathrm{V}<20$ and $0.06 \mathrm{mag}$ for $20<\mathrm{B}, \mathrm{V}<21$. Incompleteness in the data was estimated to be $30 \%$ for $20<\mathrm{V}<20.5$ and $55 \%$ for $20.5<\mathrm{V}<21$.

This data set is ideally suited for our use as it provides the most complete (to 21 mag in $\mathrm{V}$ over all the surveyed regions) census of the blue star population of NGC 6822. Wilson (1992a) has estimated the colour excess of the main sequence stars in the galaxy to be $E(B-V)=0.45 \pm 0.05$ with 0.3 mag of reddening coming from foreground sources and 0.15 mag from sources internal to the galaxy. For the purposes of our investigation, we have selected only those stars with $\mathrm{V} \leq 21$ and $B-V \leq 0.5$ mag. The colour criterion, which is based on the estimated average reddening for the galaxy, the intrinsic colour of $\mathrm{O}$ stars on the zero age main sequence $(B-V=-0.3 \mathrm{mag}$, Flower 1977) and the observed width of the main sequence, assures us that the maximum number of potential OB stars will be selected for further investigation.

\section{2. $H \alpha$ EMISSION}

We used red-continuum and $H \alpha \mathrm{CCD}$ images of the entire galaxy as well as its immediate surroundings kindly provided by P. Massey and G. Jacoby. The images were obtained using the KPNO 4-m telescope and $2048 \times 2048 \mathrm{~T} 2 \mathrm{~KB}$ chip (scale $0.48^{\prime \prime}$ pixel $^{-1}$ ) on the night of January 7, 1992. Exposure times were 60 sec for the red-continuum and $500 \mathrm{sec}$ for the $H \alpha$ frame. These frames have been trimmed and overscan subtracted, flat fielded and bias subtracted in the usual manner using the image processing package CCDPROC within IRAF. The $H \alpha$ image was scaled to the same flux level as the red-continuum image using a number of bright, unsaturated stars. As the seeing in the $H \alpha$ image was slightly worse than that in the continuum image, the latter was gaussian convolved so that stars on both images had the same full width at half maximum. The final continuum-subtracted image for NGC 6822, hereafter referred to as the $H \alpha$ image, was then obtained by subtracting the convolved red-continuum image from the $H \alpha$ image.

The $H \alpha$ image was calibrated by comparing published $H \alpha$ fluxes from Hodge et al. (1989) with the observed number of counts above the mean background for 15 bright (surface brightness $\mathrm{I} \geq 10^{-13} \mathrm{erg} \mathrm{cm}^{-2}$ $\mathrm{sec}^{-1}$ ) isolated regions in the galaxy. The slope of this relation, which is illustrated in Figure 1, determines the uncorrected calibration constant, $\gamma$. As the Hodge et al. (1989) $H \alpha$ fluxes were measured above the atmosphere, for our purposes $\gamma$ had to be corrected for foreground and internal extinction. Taking $\mathrm{A}_{H \alpha}=2.59 \times \mathrm{E}(\mathrm{B}-\mathrm{V})=1.17 \pm 0.13$ (Schild 1977), we determined the extinction corrected calibration constant, $\Gamma$, to be $6.5 \pm 0.8 \times 10^{-18} \mathrm{erg} \mathrm{cm}^{-2} \mathrm{sec}^{-1}$ count $^{-1}$. The uncertainty in $\Gamma$ was estimated at $\sim 12 \%$. This figure included the error in $\gamma$ (estimated at $\sim 2 \%$ based on the rms error in the slope of the $H \alpha$ luminosity versus count graph) as well as that in $\mathrm{A}_{H \alpha}$. In addition to the reddening, the data had to be corrected for contamination from the [NII] line at $6583 \AA$ which was also observed through the $H \alpha$ filter used. This was done by using the data of Pagel et al. (1980) that give an average $[\mathrm{NII}] / H \alpha$ ratio of $5.5 \% \pm 1 \%$ over seven H II regions in NGC 6822 . Thus scaling down all counts on the $H \alpha$ image by a factor of 0.945 corrects for the $[\mathrm{NII}]$ contamination in the data.

Motived by the investigation in M33 as well as visual inspection of the $H \alpha$ image, we separated the galaxy into four types of emission regions: bright, halo, diffuse and field. The four regions were defined by their $\mathrm{H} \alpha$ surface brightness, $I$, such that $I \geq 1.6 \times 10^{-15} \mathrm{erg} \mathrm{s}^{-1} \mathrm{~cm}^{-2} \operatorname{arcsec}^{-2}$ for the bright regions, $4.4 \times 10^{-16} \leq I<1.6 \times 10^{-15} \mathrm{erg} \mathrm{s}^{-1} \mathrm{~cm}^{-2} \operatorname{arcsec}^{-2}$ for the halo regions, $1.3 \times 10^{-16}<I \leq 4.3 \times 10^{-16} \mathrm{erg} \mathrm{s}^{-1}$ $\mathrm{cm}^{-2} \operatorname{arcsec}^{-2}$ for the diffuse regions and $I<1.3 \times 10^{-16} \mathrm{erg} \mathrm{s}^{-1} \mathrm{~cm}^{-2} \operatorname{arcsec}^{-2}$ for the field regions (see Figure 2). For comparative purposes, the dividing lines in surface brightness between the bright, halo and 
diffuse regions are consistent with those adopted in M33 (without the [NII] correction applied to the data) but that between the diffuse and field emission regions was motived by visual inspection of the $H \alpha$ image of NGC 6822. We note that the diffuse and field regions were not separated in M33 as it was not clear whether they were two distinct components of the emission in the M33 study or, rather, a single component that was smoothly declining in surface brightness. The surface brightnesses quoted above have been corrected for extinction in $H \alpha$ and are measured above an observed average sky surface brightness over the galaxy of $\mathrm{I}_{b g}=5.32 \pm 0.53 \times 10^{-16} \mathrm{erg} \mathrm{s}^{-1} \mathrm{~cm}^{-2} \operatorname{arcsec}^{-2}$. The sky emission level was obtained by averaging the modal photon counts of a number of isolated regions past the optical limits of the galaxy. As this emission was observed to be fairly uniform over the entire image, it is assumed to be "sky" and not a smooth emission component within NGC 6822. As such, it was not included in calculating the $H \alpha$ luminosity of a region. One final point to note is that although the $H \alpha$ emission in the entire galaxy is available for study, we have restricted our attention to only those regions for which BV photometric data are available i.e. the main body and northern bar regions of NGC 6822 .

\subsection{PREDICTED $H \alpha$ LUMINOSITY FROM OB STARS}

To enable a comparison of the observed $H \alpha$ fluxes with those expected from the associated stellar population, we have used the stellar ionization models of Auer \& Mihalas (1972) (hereafter A\&M), Kurucz (1979) and Panagia (1973) as well as the blackbody approximation. Given the effective temperature $\left(T_{\text {eff }}\right)$ and effective gravity $(g)$ of a star, the models predict the expected Lyman continuum flux from the star. The predicted ionizing flux is, in turn, used to obtain an $H \alpha$ luminosity by an assumed recombination scenario. The models differ in their treatment of the stellar atmospheres. In particular, the A\&M results are based on nonblanketed NLTE model atmospheres composed of hydrogen and helium only, while the Kurucz models, which include the effects of line blanketing, have LTE atmospheres of solar composition. Finally the Panagia models are based on a combination of the NLTE A\&M models and the LTE models due to Bradley and Morton (1969), Morton (1969) and Van Citters \& Morton (1970), and appropriate values of $g$ are used to calculate the flux of Lyman continuum photons as a function of $T_{\text {eff }}$ for stars of luminosity class I, III, V as well as the zero age main sequence (ZAMS).

We have used the calibration equations given in Parker \& Garmany (1993) to calculate the effective temperatures and bolometric corrections (BC) for all stars with $\mathrm{M}_{V} \leq 21$ and $B-V \leq 0.5$. As only $\mathrm{B}$ and $\mathrm{V}$ photometry is available, we have used only those equations which are independent of the reddening free index $\mathrm{Q} \equiv(U-B)-0.72(B-V)$. The effective temperature was calculated using one of equations $4 \mathrm{~b}$ to $4 \mathrm{e}$ in Parker \& Garmany (1993) while the BC of the star was determined from $T_{\text {eff }}$ using one of equations $5 \mathrm{a}$ to 5 d (ibid). Effective temperatures thus calculated were used as inputs to the ionization models while the BCs were used to estimate, where necessary, the radius of the star emitting the ionizing flux.

Details of the application of the various ionization models used here are given in Paper I. Briefly, for the blackbody as well as the A\&M and Kurucz models for various effective gravities, we have used Table XIV from Massey et al. (1989b) which neatly summarizes the expected Lyman flux at the surface of the star for $T_{\text {eff }}$ between $20,000^{\circ} \mathrm{K}$ and $50,000 \mathrm{~K}$. We have adopted $\log (g)=3.5$ for stars with $T_{\text {eff }} \leq 35,500 \mathrm{~K}$, and $\log (g)=5.0$ for stars with $T_{\text {eff }}>35,500 \mathrm{~K}$ for the Kurucz models and $\log (g)=4.0$ for the A\&M models. In order to apply the Panagia models, we were required to determine the luminosity class of the star as the models give the total ionizing flux (as a function of $T_{\text {eff }}$ ) separately for ZAMS, supergiant, class III and main sequence stars. We estimated the luminosity class of the object by comparing the model V mag, 
$\mathrm{M}_{V}^{P}$ (for a given $T_{\text {eff }}$ ), with that observed. The $\mathrm{M}_{V}^{P}$ that was closest to the observed $M_{V o}$ determined the luminosity class. All stars with $M_{V o} \leq-6.0$ were taken to be supergiants (class I).

To convert Lyman continuum photons to $H \alpha$ photons, we used the relations $\mathrm{N}(\mathrm{LyC}) / \mathrm{N}(H \alpha)=2.22$ (Osterbrock 1989) for Case B recombination, which is appropriate for optically thick gas at 10,000 K and densities of $10^{2}-10^{4} \mathrm{~cm}^{-3}$, as well as $\mathrm{N}(\mathrm{LyC}) / \mathrm{N}(H \alpha)=5.36$ (Brockelhurst 1971) for Case A recombination which is appropriate for gas at 10,000 $\mathrm{K}$ that is optically thin in all Lyman transitions. Clearly, Lyman continuum photons in optically thin gas produce fewer $H \alpha$ photons than those in optically thick gas. While Case B recombination is the most commonly used approximation in the study of $\mathrm{H}$ II regions, Case A recombination was included for stars found in the diffuse and field emission regions where the optical depth of the surrounding gas may be smaller.

\subsection{GAUGING THE RELIABILITY OF THE IONIZATION FLUX}

In order to gauge the reliability of the ionization fluxes determined from photometry plus the Panagia models, we compared the photometric spectral classes (which determine the predicted ionizing flux due to each star) for a number of stars in NGC 6822 with spectral classes obtained from optical spectroscopy. The optical spectra for 37 stars were obtained during the nights of July 19-21, 1993 using the multi-object spectrometer ARGUS on the CTIO 4-m telescope. Details of the observation, reductions and spectral classifications are described in detail in Massey et al. (1995). The stars selected for spectroscopic study were some of the brightest, blue stars from the photometric list of Wilson (1992a). Seven of the stars are found to be Galactic foreground stars (based on their spectral classification as A or G type) and 2 were identified as Wolf-Rayet star thus leaving only 28 OB type stars in NGC 6822 for which we have obtained spectral classes. Due to the small number statistics, we have not used these stars to perform a direct test of the ionization balance in the galaxy.

Although a number of the ARGUS spectra were strong enough to identify the subclass as well as the luminosity class of the object, in some cases only an approximate identification of spectral type or subclass was possible. Spectral classes determined from photometry plus Panagia models were based on the calculated $T_{\text {eff }}$ and observed $\mathrm{M}_{V}$ of the star (as in $\S 2.3$ ). As the theoretical ionizing flux models are not defined for stars of spectral classes later than B3 (or photometrically determined $T_{\text {eff }}<T_{\text {eff,L}}$ ), all such stars were left unclassified. The 7 Galactic foreground stars and Wolf-Rayet stars were included in the sample in order to test the model predictions for respectively, late type (or low $T_{\text {eff }}$ ) and evolved stars.

Before proceeding further we note that the results of this type of comparison must be treated with caution due to the limited resolution and signal-to-noise $(\mathrm{S} / \mathrm{N})$ of the observed spectra as well as the uncertainties in the colour-magnitude-ionizing flux and absolute magnitude-spectral class relations from Panagia (1973) which were used to determine the photometric classifications. The uncertainties in the spectroscopic classifications can be up to 6 classes and/or up to 2 or more luminosity classes for spectra where classification was even possible. The reason for this large an error is mainly due to the extreme distance of NGC 6822. Even with the long integration times, typically 3.5 to 4 hours per star, the S/N ratio per resolution element was 50-80. Characteristically, an $\mathrm{S} / \mathrm{N}$ ratio greater than 100 is required for good identifications. To gauge the uncertainty in the photometric spectral classification, we included the photometric errors associated with each star to the model inputs $(\mathrm{V}$ and $(\mathrm{B}-\mathrm{V})$ ) to find the average change in spectral class was $\sim 4$ classes. 
In light of the above, we find that the match between spectral classes determined from the ARGUS spectra with those from photometry+models (uniform $\mathrm{E}(\mathrm{B}-\mathrm{V})=0.45$ ) is reasonably good: ignoring the luminosity class, of the 37 stars, 19 or just over $50 \%$ of the identifications agreed or were within a few subclasses of each other. Of the 18 stars with poor matches, 16 had photometric classifications that were significantly earlier than those observed spectroscopically while the remaining 2 were classified later than those observed. The results for stars spectroscopically identified to be earlier than O4, later than B3 or Wolf-Rayet were similar: 9 out of 17 spectral classifications were in agreement.

In conclusion, it would appear that photometric classifications are slightly biased towards earlier spectral types. This trend can be explained by the presence of unresolved binary stars in the galaxy. In light of the fact that $T_{e f f}$, and hence the photometric classification of high mass stars, is much more sensitive to their $\mathrm{V}$ mag than their (B-V) colours (as the colours of massive stars on the main sequence are degenerate), an unresolved binary would be detected as a more luminous, and therefore earlier type star then the individual stars making up the binary. Furthermore, studies indicate that roughly $50 \%$ of all stars are found in binary systems (Abt 1978; Duquennoy \& Mayor 1991) which matches surprisingly well with the fraction of photometric identifications that did not agree with the spectroscopic ones. While we cannot gauge the exact quantitative effect of the photometric bias on the theoretical ionizing fluxes, we can safely say that its overall effect is to predict more flux then if spectroscopic data were used. In light of the results of this section and for the purposes of our investigation, we shall consider any agreement between observed and predicted fluxes that is within a factor of 2 to be acceptable.

\section{IONIZED GAS AND OB STAR DISTRIBUTION}

To study the common assumption that $\mathrm{O}$ and $\mathrm{B}$ stars are predominantly found in bright $\mathrm{H}$ II regions, we looked at the distribution of these stars as a function of their surrounding ionized gas environment. We identified the ionized gas environment of a star as either bright, halo, diffuse, or field according to the average $H \alpha$ surface brightness inside a circle of radius of 3 pixels $(\sim 3.5 \mathrm{pc})$ centered on the star. The distribution of the OB stars (as a function of their visual magnitude) within the four ionized gas environments is summarized in Table 1.

It is immediately clear that OB stars are not all located in the bright $\mathrm{H}$ II regions: in fact, $\sim 50 \%$ of all the stars with $\mathrm{V}<21$ and $(\mathrm{B}-\mathrm{V})<0.5$ are found in the field (see Figure 3). Moreover, field stars outnumber those in the bright regions by a factor of three. This trend is also suggested by the brightest stars in the survey where slightly less than half of all stars with $\mathrm{V} \leq 18$ are seen to be in the field while only a quarter are in the bright regions. At $\sim 11 \%$, the halo houses the smallest percentage of OB stars in the galaxy while the diffuse emission region carries a substantial $24 \%$ of the total. We see faint stars dominate all four regions and that percentage of these stars within the different regions is comparable.

We can now determine the important quantity, the fraction of the main sequence lifetime of an $\mathrm{O}$ star that is spent within a recognizable $\mathrm{H}$ II region, defined as $f_{H I I} \equiv\left(N_{B}+N_{H}\right) / N_{T O T}$ where $N_{T O T}$ is the total number of stars in the sample and $N_{B}$ and $N_{H}$ are the numbers of stars in, respectively, the bright and halo regions of the galaxy. By defining a recognizable H II region to be composed of either a bright or halo emission region, we are assured of obtaining the most conservative estimate of this key quantity. From Table 1 , we find $f_{H I I}=0.29$, implying that roughly $70 \%$ of the main sequence lifetime of an OB star is spent outside of a recognizable H II region. The consequence of this result to the lifetimes of molecular clouds is 
that if OB stars escape from bright and halo H II regions by destroying their parent molecular clouds, the molecular cloud lifetimes after forming OB stars could be as short as $\sim 1-3 \times 10^{6}$ yrs (or $1 / 3$ the typical main sequence lifetimes of $3-8 \times 10^{6} \mathrm{yrs}$ for $120-20 \mathrm{M}_{\odot}$ stars assuming $\mathrm{Z}=.020$, Schaller et al. 1992).

We summarize in Table 2 a few important properties of the ionized gas environments and the associated OB star distribution. A striking point to note is that while the surface density of stars in the bright region is a factor of $\sim 6$ times greater than that in the field, the field occupies an area that is $\sim 17$ times that of the bright region. These two results provoke the puzzling question of whether the field stars could have originated in and percolated out of existing bright ionized gas environments. Citing a study of the Trapezium cluster in Orion by Zuckerman (1973), Churchwell (1991) suggests a typical O star velocity relative to its parent molecular cloud is $3 \mathrm{~km} \mathrm{~s}^{-1}$. At this speed, an $\mathrm{O}$ star could travel $\sim 30$ pc over its lifetime of $\sim 10$ Myr. This scales falls far short of the characteristic distances ( 200-500 pc) of the large field regions and, therefore, field stars cannot all have simply percolated out of the H II regions currently visible.

Also from Table 2 we find that the relative total $H \alpha$ luminosity per field star is $\sim 4 \%$ that of a star in a bright H II region, which raises the question of whether the field is "leaking" Lyman continuum photons that presumably escape from the galaxy. An alternative possibility is that the field population is older than that in bright H II regions so that fewer early type stars with large ionizing fluxes still remain. One way to investigate this possibility is to look at the bright star distribution, which is most sensitive to age differences, in the different ionized gas environments. The distribution of stars brighter than $\mathrm{V}=18$, normalized to the total number brighter than $\mathrm{V}=19$, in the bright, halo, diffuse and field regions are, respectively, $7 \% \pm 4 \%$, $1 \% \pm 1 \%, 5 \% \pm 3 \%$ and $12 \% \pm 5 \%$. Clearly, there is no firm indication that any one of the ionized gas environments has an over-abundance of bright stars and therefore there is no evidence for age segregation between the different regions. The slope of the stellar luminosity function can be another indicator of an age difference: shallow slopes imply younger stars dominate the region while steep slopes indicate older populations dominate. Plots of the luminosity functions, in terms of $\mathrm{M}_{V}$ versus $\log \left(\mathrm{N}_{*}\right)$ where $\mathrm{N}_{*}$ is the total number of stars in the magnitude bin (width $0.5 \mathrm{mag}$ ), are given in Figure 4. Weighted least-squares fits to the data to a limiting $\mathrm{V}$ magnitude of 19 for the bright region (to reduce the effects of incompleteness) and $\mathrm{V}=19.5$ for all others yield slopes of $0.36 \pm 0.05$ (bright), $0.37 \pm 0.19$ (halo), $0.53 \pm 0.16$ (diffuse), 0.47 \pm 0.07 (faint) and $0.53 \pm 0.05$ (entire image). Clearly there is no evidence for significant differences in the luminosity function slopes and therefore, no indication that the stellar populations of the four H II environments have different average ages. As such, it appears that the differences in the $H \alpha$ luminosity per $\mathrm{O}$ star in the field compared to that in the bright regions indicates a difference in the gas environment of the $\mathrm{O}$ stars and may indicate that ionizing radiation from field stars is escaping out of the galaxy.

\section{IONIZATION BALANCE}

In this section, we test one of the fundamental assumptions of star formation rate calculations from $H \alpha$ luminosities, namely that the region under consideration is ionization bounded i.e. the Lyman continuum flux emitted by the stars remains in the region. This is accomplished by comparing the observed $H \alpha$ emission from stars with that predicted from stellar ionization models. Although the detailed analysis is based on the models of Panagia (1973), we have also used the blackbody, Kurucz, and A\&M models for comparative purposes as well as to serve as a check on the Panagia results. 
In all applications of the ionizing flux models, we have followed a method similar to that described in Paper I. In brief, for comparative purposes, we have imposed an upper and lower limit to $T_{e f f}$, $T_{e f f, U}=60,000 \mathrm{~K}$ and $T_{e f f, L}=30,000 \mathrm{~K}$. The lower limit $T_{\text {eff,L }}$ is chosen as the minimum effective temperature for which all ionization models are defined. Although all four models are defined up to $T_{\text {eff }}=50,000 \mathrm{~K}$ (for an $\mathrm{O} 4$ class $\mathrm{V}$ star), we have chosen $T_{\text {ef } f, U}=60,000 \mathrm{~K}$ so as to provide a small margin of error at the high $T_{\text {eff }}$ end as well as to roughly account for the Lyman continuum flux for stars earlier than O4. Stars that had calculated effective temperatures between 50,000 K and 60,000 K were all assigned Lyman fluxes corresponding to $50,000 \mathrm{~K}$ from the models. Stars with $T_{\text {eff }}<T_{\text {eff,L }}$ and $T_{\text {eff }}>T_{\text {eff,U }}$ are assigned zero flux. Note that we have not attempted to account for the ionizing flux from stars with effective temperatures outside these limits: stars with $T_{\text {eff }}<T_{\text {eff,L }}$ are most likely to be evolved late type stars with very small contributions to the total Lyman continuum luminosity while those with $T_{\text {eff }}>T_{\text {eff,U }}$ would not only entail extrapolating the $T_{\text {eff }}$-Lyman continuum flux relations to highly uncertain limits, but more importantly, such high effective temperatures may be a result of photometric errors rather than reflecting the true $T_{\text {eff }}$ of the star (for example, Massey et al. (1989a, 1989b) have found many stars with unrealistic (B-V) or (U-B) colours which they attributed to photometric errors). Finally, we did not consider the presence of Wolf-Rayet stars in the galaxy as the ionizing flux from these stars, while likely to be very high, is rather uncertain (Massey et al. 1989b). The effect of accounting for Wolf-Rayet stars in NGC 6822 would be to increase the predicted ionizing flux.

Assuming Case B recombination, the $H \alpha$ flux from all stars with $T_{\text {eff }, L}<T_{\text {eff }}<T_{\text {eff,U }}$ predicted by the four different ionization models (in units of $10^{39} \mathrm{erg} \mathrm{s}^{-1}$ ) is: 10.7 (Panagia), 7.2 (blackbody), 5.7 (Kurucz) and 6.8 (A\&M). The highest (Panagia) and lowest (Kurucz) estimates differ by a factor of roughly 2. Considering the differences in the models and the arguments of $\S 2.4$, the agreement between them is acceptable. In the remainder of the section we will restrict our discussion to the results obtained using the Panagia models only, keeping in mind that the corresponding results from the other models scale as above.

As the minimum effective temperature for which the Panagia models are defined depends on the luminosity class of the star, i.e. $T_{e f f}=15100 \mathrm{~K}$ (for class I), $T_{e f f}=16,900 \mathrm{~K}$ (for class V and ZAMS) and $T_{e f f}=16,000 \mathrm{~K}$ (for class III), we have defined for each luminosity class the corresponding $T_{e f f, L}$ inferred from the models. Of the 724 stars for which we had photometry, 428 had effective temperatures between $T_{e f f, L}$ and $T_{e f f, U}$. The percentage of stars with $T_{\text {eff }}<T_{\text {eff,L }}$ or $T_{\text {eff }}>T_{\text {eff,U }}$ (and therefore for which no ionizing flux could be assigned) was $58 \%(\mathrm{~V}<18), 28 \%(18<\mathrm{V}<19), 27 \%(19<\mathrm{V}<20)$ and $48 \%(20<\mathrm{V}<21)$. As such, it would appear that a significant fraction of the stars are not contributing to the total $H \alpha$ luminosity observed.

Photometric incompleteness for all stars with $20<\mathrm{V} \leq 21$ as well as incompleteness due to stars fainter than the survey limit of $21 \mathrm{mag}$ have also been accounted for. To correct for the photometric incompleteness of the data, we have multiplied the predicted fluxes by a factor of 1.43 for $20<\mathrm{V} \leq 20.5$ and 2.22 for $20.5<\mathrm{V} \leq 21$ where the correction factors follow from the estimated incompleteness of $\sim 30 \%$ (for $20<\mathrm{V} \leq 20.5$ ) and $\sim 55 \%$ (for $20.5<\mathrm{V} \leq 21$ ) (Wilson 1992a). In order to correct for stars with $\mathrm{V}>21$, we have used a method similar to the one used in the study of M33 (Paper I). Briefly, we calibrated a main sequence mass- $\mathrm{M}_{V}$ relation (using theoretical stellar evolution tracks for $\mathrm{Z}=0.020$ from Schaller et al. (1992)) to find that, at the distance and reddening of NGC $6822, \mathrm{~V}=21$ corresponds to $18 \mathrm{M}_{\odot}$ or roughly an O9.5 to B0 star (Popper (1980) and Schmidt-Kaler (1982)). We then used the Salpeter initial mass function $N(m) d(M)=A m^{\alpha} d m(\alpha=2.35$, Salpeter 1955) to calculate the total number of stars for each spectral (sub)class from O9.5 to B3 (the latest class for which the Panagia models give theoretical fluxes). For each of the four ionized gas environments, a different normalization constant $A$ was determined by equating the 
integral of the initial mass function between $18 \mathrm{M}_{\odot}$ and $65.5 \mathrm{M}_{\odot}$ to the total number of stars in the region for which we could determine $T_{\text {eff }}$ (either uncorrected for the conservative flux estimate, or corrected for incompleteness for the best flux estimate). With $A$ in hand, we then calculated the total ionizing flux in each spectral class (or subclass) bin between O9.5 and B3 by multiply the average theoretical flux for that bin (class $\mathrm{V}$ assumed) by the total predicted number of stars in the bin. The correction to the flux from stars fainter than $\mathrm{V}=21$ was then simply the sum of the ionizing flux from the individual bins.

Observed and theoretical fluxes from stars in the four ionized gas environments corrected, in turn, for the various effects described above, are summarized in Table 3. The theoretical fluxes are organized in the following manner: (1) no corrections for incompleteness (minimum flux case); (2) corrected for incompleteness for stars brighter than $\mathrm{V}=21$; (3) corrected for incompleteness for $\mathrm{V} \leq 21$ and $\mathrm{V}>21$ (best estimate) and (4) corrected for stars below the survey limit (i.e. $\mathrm{V}>21$ ).

The best overall agreement between theory and observation is obtained for the case where the data are uncorrected for any incompleteness. Over the total image, the two differ by a factor of 1.8 (Case A used in the diffuse and field) and $\sim 2.8$ (Case B used everywhere). The predicted flux exceeds that observed by a factor of 1.4 in the bright, 2.1 in the halo, 1.1 in the diffuse (2.8 for Case B recombination) and 5.9 in the field (14 for Case B recombination). While the bright, halo and diffuse regions appear to be relatively close to ionization balance, the field is clearly not in ionization balance. Since $50 \%$ of the blue stars in NGC 6822 are found in the field, up to $50 \%$ of the ionizing photons produced may be escaping from the galaxy.

The effect of correcting the data for $\mathrm{V}>21$ incompleteness is minor: the minimum flux estimates for the four regions, separately and combined, are increased by roughly $1 \%$ when the flux expected from stars fainter than the survey limits are included. In contrast, correcting the data for photometric incompleteness for $20<\mathrm{V} \leq 21$ increases the predicted flux from the uncorrected, uniform reddening case by a factor of $\sim 1.4$. The total theoretical flux, after this correction has been made, exceeds that observed by a factor of 2.0 in the bright, 2.9 in the halo, 1.6 in the diffuse (4.0 for Case B), 8.0 in the field (19 for Case B), and 2.5 for the entire galaxy (3.8 for Case B used everywhere). Clearly, of all the different corrections, only that of photometric incompleteness for stars with $20<\mathrm{V} \leq 21$ is significant. It is reassuring that the more uncertain, model dependent correction for stars with $\mathrm{V}>21$ is unimportant compared to the simpler and more easily measured photometric correction.

The best estimate of the predicted flux corrects for photometric incompleteness over $20<\mathrm{V} \leq 21$ and includes flux from main sequence stars fainter than $\mathrm{V}=21$. Over the entire surveyed region, these fluxes are a factor $\sim 2.5$ (Case A) to $\sim 3.9$ (Case B) greater than observed. The best case for ionization balance is in the diffuse and bright emission regions where the predicted fluxes are, respectively, $\sim 1.7$ and $\sim 2.0$ (assuming Case A recombination) times those observed. The worst case for ionization balance is in the field, where predicted and observed fluxes for Case A and B differ from that observed by factors of, respectively, $\sim 8$ and $\sim 20$. On average, the best estimate fluxes are approximately 1.4 times the corresponding conservative estimates.

It is clear from Table 3 that the predicted and observed fluxes in the diffuse and field emission regions are best matched when Case A (optically thin) recombination is assumed. That the gas density in these regions should be lower than that in the bright and halo regions is not unreasonable. While we have no direct measure of the gas density in the different ionization regions, we can place limits on this quantity by comparing the observed $H \alpha$ surface brightness with that expected for an H II region in gas of known

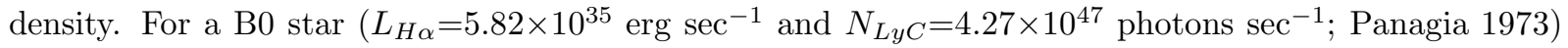
located in the diffuse emission region, the maximum surface brightness observed $\left(\mathrm{I}_{F}=4.32 \times 10^{-16} \mathrm{erg}\right.$ 
$\mathrm{s}^{-1} \mathrm{~cm}^{-2}$ arcsec ${ }^{-2}$ ) limits the gas density to be $\leq 5 \mathrm{~cm}^{-3}$. In obtaining the above result, we have used the Stromgren radius (eg. Spitzer 1978) to estimate the radius of the H II region and assumed the gas within the Stromgren sphere of the star is completely ionized. Similarly, if the ionizing source is an O5 star, the gas density in the diffuse region would have to be $\leq 0.5 \mathrm{~cm}^{-3}$ for the surface brightness of the $\mathrm{H}$ II region to match the maximum level observed. As the maximum surface density in the field is lower than that in the diffuse region, the field gas density would be correspondingly smaller. If the surface brightnesses of $\mathrm{H}$ II regions formed by $\mathrm{B} 0$ and $\mathrm{O} 5$ stars are to equal the average surface brightness observed in bright $\mathrm{H}$ II regions $\left(\mathrm{I}_{B}=4.11 \times 10^{-15} \mathrm{erg} \mathrm{s}^{-1} \mathrm{~cm}^{-2} \operatorname{arcsec}^{-2}\right.$ ), the gas density must be $\sim 15 \mathrm{~cm}^{-3}$ (B0) and $\sim 1 \mathrm{~cm}^{-3}$ (O5). Thus the gas density in the diffuse and field regions is likely to be lower than that in the bright $\mathrm{H}$ II regions which lends some support to our assumption of Case A recombination in the diffuse and field regions and Case B elsewhere in the galaxy.

What are the implications of the above findings to the determination of high mass SFRs from $H \alpha$ luminosities? Recalling from our earlier discussion that the SFR scales with the Lyman continuum luminosity, we would expect the SFR in NGC 6822 to underestimate the "true" SFR i.e. that which would be measured if all the stellar ionizing radiation is converted into, and observed as, $H \alpha$ emission. Over the entire galaxy, the difference between the observed and true SFR is a factor of $\sim 2-4$. Moreover, while the difference is less than a factor of 4 in the bright, halo and diffuse regions, in the field the discrepancy between the observed and true SFR is a factor of $\sim 6-20$. Clearly, this technique to obtain the rate of formation of massive stars is sensitive to the stellar environment and cannot be used indiscriminately in any environment. For the field region, which houses a significant $\sim 50 \%$ of the total number of OB stars in the galaxy but is losing the greatest amount of ionizing flux, this technique is most unreliable. Our results also illustrate the importance of choosing a recombination scenario that is appropriate to the region under investigation.

Assuming Case B recombination when Case A is more likely to be appropriate systematically underestimates the SFR. This may be a problem for many SFRs that are determined using Kennicutt's (1983) relation which assumes Case B recombination over the entire galaxy. In NGC 6822, the SFR obtained by applying the Kennicutt formula to the observed $H \alpha$ luminosities in all four ionized gas regions $\left(\mathrm{SFR}_{K}\right)$ underestimates by $\sim 30 \%$ the SFR obtained when Case A is used in the field and diffuse regions. Moreover, by taking the flux lost from the field region into account (by assuming the actual $H \alpha$ luminosity in the field is the minimum flux estimated from the Panagia ionization models and Case A recombination in the diffuse and field regions) we find $\mathrm{SFR}_{K}$ underestimates the true SFR by $\sim 50 \%$.

\subsection{IONIZATION BALANCE IN HUBBLE I AND III}

What is the state of ionization balance on a small scale? To answer this question we focus on the bright H II knot consisting of Hubble I and III located in the northern bar of the galaxy (see Figure 5). First identified by Hubble in 1925, Hubble I and III collectively encompass an area roughly $0.3 \mathrm{kpc}^{2}$ (for $\left.\mathrm{I} \geq 1.3 \times 10^{-16} \mathrm{erg} \mathrm{s}^{-1} \mathrm{~cm}^{-2} \operatorname{arcsec}^{-2}\right)$. This region was among the many H II regions of NGC 6822 studied by Hodge \& Lee (1989) who measured the $H \alpha$ luminosity of the region to be $6.21 \times 10^{-16} \mathrm{erg} \mathrm{s}^{-1} \mathrm{~cm}^{-2}$. We have summarized in Table 4 the observed and predicted $H \alpha$ emission in the bright, halo and combined (bright and halo) components of Hubble I and III. The theoretical fluxes are calculated by applying the models of Panagia (1973) to the 43 stars in the region for which we have photometry. In all cases, the models predict more flux than observed in the bright emission region and less flux than observed in the 
halo region. Moreover, the agreement between theory and observations is very good (within a factor of 1.7 to 2.3 ) in the bright regions but rather poor in the halo (between a factor of 0.07 to 0.16 ). This large discrepancy in the halo region is most likely due to the very few (only 3 ) stars located there. As it turns out, the flux measured and predicted in the halo is almost negligible when compared to that in the bright region and so does not affect the overall conclusions of ionization balance in this individual region. Concentrating on the combined (bright and halo) regions, we find that the predicted and observed fluxes are best matched when the data are left uncorrected where the two differ by a factor of $\sim 1.6$. Correcting for incompleteness $(\mathrm{V} \leq 21)$ has the greatest overall effect on the predicted fluxes which are seen to increase by a factor of $\sim 0.4$ from the minimum flux case and are a factor of $\sim 2.2$ greater than that observed. Finally, the best estimate case which corrects for incompleteness above and below $\mathrm{V}=21$, yields predicted fluxes that are slightly more than twice those observed and therefore within the allowed range of uncertainty. Based on these results, we conclude that Hubble I and III are collectively likely to be in ionization balance.

\section{COMPARISON WITH M33}

Having investigated the ionized gas-OB distribution as well as considered the question of ionization balance in NGC 6822, we can now address the question of how the results for this dwarf irregular system compare with those found for the spiral galaxy M33. In order to do so, it is important that the colour and brightness cutoffs in the two galaxies are matched so that the same types of OB stars are sampled. In the case of $\mathrm{M} 33$, only those stars with $\mathrm{V} \leq 21$ and $(\mathrm{B}-\mathrm{V}) \leq 0.4$ were selected for study. Assuming a total reddening of $\mathrm{E}(\mathrm{B}-\mathrm{V})=0.3 \mathrm{mag}$ (Wilson 1991), and a distance of $0.79 \mathrm{Mpc}$ to the galaxy (van den Bergh, 1991), the absolute magnitude cutoff corresponding to the limit of $\mathrm{V}=21$ is $\mathrm{M}_{V}=-4.4$ mag. At the NGC 6822 distance and adopted reddening of $\mathrm{E}(\mathrm{B}-\mathrm{V})=0.45$, the selection criterion for matching the two galaxies is $\mathrm{V} \leq 20.5$ and $(\mathrm{B}-\mathrm{V}) \leq 0.55$.

Although the diffuse emission component was not differentiated from the field component in the M33 investigation (Paper I), we have, for sake of comparison, done so here (see Figure 6). The limiting surface brightness separating the field and diffuse regions in M33, $I_{F D, M 33}$, is chosen to match that in NGC 6822 before correcting for [NII] emission so that $I_{F D, M 33}=1.3 \times 10^{-16} \mathrm{erg} \mathrm{s}^{-1} \mathrm{~cm}^{-2} \operatorname{arcsec}^{-2}$ uncorrected and $I_{F D, M 33}=1.03 \times 10^{-16} \mathrm{erg} \mathrm{s}^{-1} \mathrm{~cm}^{-2} \operatorname{arcsec}^{-2}$ corrected fro [NII] emission. A number of interesting features of the ionized gas distributions are revealed on comparing Figures 3 and 6 . Unlike NGC 6822, where the diffuse emission is extended over the galaxy, in M33 the diffuse gas appears to be confined to a narrow region around the halo emission, thus giving the impression that the diffuse gas is, in fact, a thin "halo" of the latter. Additionally, the distinction between the bright and halo regions in M33 is much clearer than that in NGC 6822: while the halos in M33 are broad, extended regions, those in NGC 6822 are narrow rings or bands confined around the bright emission regions. The field regions in both galaxies are similar in that the emission is highly uniform and low level. It appears therefore that although M33 and NGC 6822 both contain bright, halo, diffuse and field emission regions, the spatial distribution of these components are not the same in the two galaxies. The main difference is seen in the halo and diffuse emission regions that appear to have exchanged roles between M33 and NGC 6822.

In Table 5 we summarize the distribution of the brightest OB stars $\left(M_{V} \leq-7.45\right.$ corresponding to $\mathrm{V} \leq 18$ in M33 and $\mathrm{V} \leq 17.5$ in NGC 6822) and total number of OB stars down to the M33 survey completeness limit

$\mathrm{V} \leq 21$ or $M_{V} \leq-4.45$ (corresponding to $\mathrm{V} \leq 20.5$ in NGC 6822 ). Considering the sample of stars brighter than $M_{V}<-4.45$, the results in the two galaxies are well matched in the bright and diffuse emission components 
( $\sim 18 \%$ (M33) and $\sim 17 \%$ (NGC 6822 ) are located in the bright and $\sim 24 \%$ (M33) and $\sim 25 \%$ (NGC 6822 ) in the diffuse) but somewhat different in the halo and field regions ( $\sim 32 \%$ (M33) compared to $\sim 11 \%$ (NGC 6822) in the halo and $\sim 27 \%$ (M33) compared to $~ 46 \%$ (NGC 6822) in the field). NGC 6822 has a proportionately larger field population then M33. Due to the significant percentage of halo stars in M33, the fractional lifetimes of an $\mathrm{O}$ star spent in a recognizable $\mathrm{H}$ II region, and thus in its parent molecular cloud, is greater in M33 $\left(f_{H I I} \sim 50 \%\right)$ than in NGC $6822\left(f_{H I I} \sim 28 \%\right)$.

What might the value of $f_{H I I}$ be telling us about molecular clouds in M33 and NGC 6822? If in both galaxies OB stars escape from bright and halo H II regions by destroying their parent molecular clouds, then molecular cloud lifetimes after the formation of OB stars are shorter in NGC 6822. This could be explained if the molecular clouds in NGC 6822 are smaller on average than those in M33, since smaller clouds would be easier to destroy. It could also be that the star formation efficiency (defined as $\mathrm{SFE}=\mathrm{M}_{*} /\left(\mathrm{M}_{*}+\mathrm{M}_{\text {gas }}\right.$ ) where $M_{*}$ and $M_{\text {gas }}$ are respectively the mass in stars and gas in the cloud) is higher in NGC 6822 so that more stars are formed and, therefore, the probability of destroying or dissipating the natal molecular clouds is much increased. Alternatively, if OB stars simply move out of their parent molecular clouds and H II regions, then the quantity $f_{H I I}$ does not imply a molecular cloud lifetime but suggests that perhaps molecular clouds are smaller in NGC 6822 than M33. Evidence that molecular clouds may be smaller in dwarf irregular galaxies than in spirals comes from a recent CO study of the SMC (Rubio et al. 1993).

Inspection of Table 6 reveals a number of interesting results from the ionization balance calculations in the two galaxies. Based on the simple minimum flux case, the bright, halo and diffuse emission regions, as well as the entire surveyed regions of both galaxies are likely to be ionization bounded. This is also suggested by the conservative and best estimate of the ionizing flux in NGC 6822. The problem area in the minimum case, as in all the others, is the field which appears to be leaking the most Lyman photons: from $\sim 4$ (in NGC 6822) and 17 (in M33) times the observed flux in the uncorrected, minimum estimate case to $\sim 5$ (in NGC 6822) and a staggering $~ 88$ (in M33) times the observed flux in the best estimate case. The field, therefore, does not appear to be in a state of ionization balance in either galaxy and furthermore, the problem is much more severe in M33 than NGC 6822. The fact that even in the minimum flux case (where no corrections for incompleteness have been made) the field stars in M33 are producing so much more ionizing flux than observed suggests that the field is truly leaky to Lyman continuum photons. Although NGC 6822 has a higher percentage of field stars contributing to the ionizing flux (i.e. stars with $\mathrm{T}_{\text {eff }, U}<T_{\text {eff }}<\mathrm{T}_{\text {eff,L }} \sim 60 \%$ in NGC 6822 and $\sim 45 \%$ in M33), it is still more effective at holding on to the ionizing photons produced by these stars than M33. This is also seen to be the case in the other individual ionized gas environments as well as the entire surveyed regions of the galaxies as a whole: for both the conservative and best estimate cases, the difference between the predicted and observed $H \alpha$ fluxes in the bright, halo, diffuse and collective regions are consistently higher in M33 than NGC 6822, which thus implies that the inner kiloparsec of M33 is in a more serious state of ionization imbalance than NGC 6822. All three models support the finding that the likelihood of ionization balance decreases in going from the bright to faint emission regions. Finally, while NGC 6822 as a whole appears to be in a state of ionization balance, the surveyed inner $1 \mathrm{kpc}$ region of M33 is clearly not.

In conclusion, it would appear that the morphological class of the galaxy may be an important factor in these types of studies. The fact that M33 appears to be so much less effective at holding on to the ionizing radiation than NGC 6822 suggests that the shapes of the galaxy are important: the flat discs of spiral galaxies may not provide a thick enough layer of gas to capture all or most of the Lyman continuum photons emitted by the OB stars, especially from those stars in the field. The surface density of stars may also be an important factor in the extent of ionization imbalance in that for two areas of similar column 
densities of neutral gas, the one with a larger surface density of OB stars may have much less gas left to ionize and would therefore be more likely to be leaking ionizing photons then one that has fewer stars per unit area. As an example consider the field regions in M33 and NGC 6822: the M33 field star surface density is twice that of NGC $6822\left(\sim 416 \mathrm{stars} \mathrm{kpc}^{-2}\right.$ compared to $\left.\sim 215 \mathrm{stars} \mathrm{kpc}^{-2}\right)$. Given that the average H I column density in these two galaxies is of the order of $\sim 10^{20} \mathrm{~cm}^{-2}$ (Deul \& van der Hulst 1987; Skillman 1987), this would mean that M33 should be twice as efficient at losing ionizing flux as NGC 6822. i.e. we would expect the ratio of the predicted to observed $H \alpha$ luminosities in the field regions of M33 to be a factor of two larger then that in NGC 6822. While what we observe is more than this (in the minimum flux case, M33 is 17.09/4.07 4.2 times more effective at losing ionizing flux than NGC 6822), the general outcome is clear: based on the surface density of OB stars, M33 can be expected to be more susceptible to leaking ionizing photons then NGC 6822.

\section{CONCLUSIONS}

We have investigated the distribution of $\mathrm{H}$ II regions and OB stars and tested the hypothesis of ionization balance within NGC 6822 using $H \alpha$ data and BV photometry of blue stars. We divide the $H \alpha$ emission in NGC 6822 into four distinct components, denoted bright, halo, diffuse and field, based on the surface brightness of the gas. The major findings of our study are summarized below.

(1) The distribution of $\mathrm{OB}$ stars brighter than $\mathrm{V}=21$ within the bright, halo, diffuse and field regions is such that $\sim 16 \%$ are located in the bright, $\sim 10 \%$ in the halo, $\sim 24 \%$ in the diffuse and $\sim 49 \%$ in the field regions. Combining the bright and halo regions reveals that only $1 / 4$ of the blue stars are found in the optically prominent H II regions. These results suggest that roughly $3 / 4$ of the main sequence lifetime of an $\mathrm{O}$ star is spent outside of such H II regions.

(2) The OB star and H II region distributions imply that if OB stars destroy their parent molecular clouds while escaping their H II regions, then molecular cloud lifetimes after the formation of OB stars must be shorter than $\sim 1-3 \times 10^{6}$ yrs. Alternatively, if the stars escape the H II regions without destroying their parent molecular clouds, then molecular cloud lifetimes could be much longer.

(3) Comparing the spectral classes determined from photometry with those deduced from optical spectroscopy shows that the former are biased towards earlier spectral types, perhaps due to unresolved binaries. Due to this effect, the theoretically predicted $H \alpha$ fluxes are likely to be over-estimated. We therefore consider any ionization balance calculations that agree to within a factor of two to be in acceptable agreement.

(4) Comparing the observed $H \alpha$ luminosities in the four ionized gas environments both separately and collectively with those predicted theoretically we find that the models consistently predict more flux than observed. The bright regions are likely to be ionization bounded as the predicted fluxes are, at most, a factor of 2 larger than observed. The halo and diffuse regions appear to be at the limit of ionization balance as the predicted fluxes can differs from that observed by up to a factor of, respectively, $\sim 3$ and $\sim 4$. In the field however, the observations and model predictions differ by a factor of $\sim 6-15$ (assuming Case A and B recombination) for the conservative case and $\sim 8-20$ for the best estimate case. This region is clearly not in ionization balance and thus fully $50 \%$ of the blue stars in NGC 6822 may be losing most of their ionizing radiation to interstellar space. 
(5) In determining a SFR from the $H \alpha$ luminosity in a galaxy, the assumption is made that the galaxy is ionization bounded and that Case B recombination is appropriate everywhere. For NGC 6822, our results show that the true SFR would be underestimated by $\sim 50 \%$ due to the leakiness of the field and the use of Case A recombination in the diffuse and field regions.

(6) Significant differences between the distributions of the OB stars within the ionized gas environments as well as the state of ionization balance in M33 and NGC 6822 suggest that the morphological class of the parent galaxy may be an important factor in the state of ionization balance of the gas and therefore in determining the SFR from the $H \alpha$ luminosity. The field in the inner kiloparsec of M33 appears to be in a more serious state of ionization imbalance than NGC 6822: M33 is losing at least $\sim 17$ times the observed $H \alpha$ flux while the figure for the field in NGC 6822 is $\sim 4$. The difference in the number of ionizing photon leaking from the two galaxies may be due to the surface density of OB stars, which is higher in M33:

since the two galaxies have comparable H I column densities, the likelihood of losing ionizing photons is increased when the stellar surface density is increased. Based on the ionization balance results, SFRs from $H \alpha$ luminosities are less likely to reflect the true SFR in the central region of M33 than in NGC 6822. 


\section{REFERENCES}

Abt, H.A. 1978 in Protostars and Planets, ed. T. Gehrels (Univ. of Arizona Press:Tucson), p. 323

Armandroff, T.E. \& Massey, P. 1985 ApJ, 291, 685

Armandroff, T.E. \& Massey, P. 1991 AJ, 102, 972

Auer, L.H. \& Mihalas, D. 1972, ApJS, 24, 193

Blitz, L. \& Shu, F. 1980 ApJ, 235, 148

Brandley, P.T. \& Morton, D.C. 1969, ApJ, 156, 687

Brockelhurst, M. 1971, MNRAS, 153, 471

Churchwell, E. 1991, in the Physics of Star Formation \& Early Stellar Evolution, ed. C. Lada \& N.D. Kylafis (Dordrecht:Kluwer), p. 221

Deul, E.R. \& van der Hulst, J.M. 1987, AAS., 67, 509

Duquennoy, A. \& Mayor, M. 1991, A\&A, 248, 485

Elmegreen, B.G. 1991, in The Physics of Star Formation \& Early Stellar Evolution, Ed. C.J. Lada \& N.D. Kylafis (Dordrecht:Kluwer), p. 35

Flower, P.J. 1977, A\&A, 54, 31

Hodge, P.W. 1977, APJS, 33, 69

Hodge, P.W. 1980 ApJ, 241, 125

Hodge, P.W., Lee, M.G. \& Kennicutt, R.C. 1988 PASP, 100, 917

Hodge, P.W., Lee, M.G. \& Kennicutt, R.C. 1989 PASP, 101, 32

Hodge, P.W., Smith, T., Eskridge, P., Macgillivray, H.T. \& Beard, S. 1991, ApJ, 379, 621

Humphreys, R. M. 1980, ApJ, 238, 65

Kennicutt, R.C. 1983, ApJ, 272, 54

Kurucz, R.L. 1979, ApJS, 40, 1

McAlary, C.W., Madore, B.F., McGonegal, R., McLaren, R.A. \& Welch, D.L. 1983, AJ, 273, 539

Massey. P., Garmany, C.D., Silkey, M. \& Degioia-Eastwood, K. 1989a, AJ, 97, 107

Massey. P., Parker, J.W. \& Garmany, C.D. 1989b, AJ, 98, 1305

Massey. P., Lang, C.C., Degioia-Eastwood, K. \& Garmany, C.D. 1994, ApJ, in press

Massey. P., Armandroff, T.E., Pyke, R., Patel, K. \& Wilson, C.D. 1995, AJ, submitted

Morton, D.C. 1969, ApJ, 158, 629

Osterbrock, D.E. 1989, Astrophysics of Gaseous Nebulae and Active Galactic Nuclei (Mill Valley: University Science Books)

Ohta, K., Tomita, A., Saito, M., Sasaki, M. \& Nakai, N. 1993, PASJ, 45, L21

Pagel, B.E.J., Edmunds, M.G. \& Smith, G. 1980, MNRAS, 193, 219

Panagia, N. 1973, AJ, 78, 929

Parker, J.W. \& Garmany, C.D. 1993, AJ, 106, 1471 
Patel, K. \& Wilson, C.D. 1995 (Paper I), ApJ, submitted

Popper, D. 1980, ARA\&A, 18, 115

Rand, R.J., Kulkarni, S.R. \& Hester, J.J. 1990, ApJ, 352, L1

Rubio, M., Garay, G., Montani, D. \& Thaddeus, D. 1993, A\&A, 271, 7

Salpeter, E.E. 1955, ApJ, 129, 608

Schaller, G., Schaerer, D., Meynet, G., Maeder, A. 1992 A\&AS, 96, 296

Schild, R. 1977 AJ, 82, 337

Schmidt-Kaler, Th. 1982, in Landolt-Bornstein: Numerical Data and Functional Relationships in Science and Technology, New Series, Vol 2, Astronomy and Astrophysics, eds K. Schaiffer and H.H. Voigt (Berlin: Springer-Verlag), p. 1

Skillman, E. 1987, in Star Formation in Galaxies, NASAP-2466, ed. C. L. Person (Washington:NASA), p. 263

Spitzer, L. 1978, Physical Processes in the Interstellar Medium (New York:Wiley)

Van Citters, G.W. \& Morton, D.C. 1970, AJ, 161, 695

Van den Bergh, S. 1991, PASP, 103, 609

Wilson, C.D. 1990, Ph.D. thesis, Caltech

Wilson, C.D. 1991, AJ, 101, 1663

Wilson, C.D. 1992a, AJ, 104, 1374

Wilson, C.D. 1992b, ApJ, 391, 144

Wilson, C.D. 1994, AJ, 434, L11

Zuckerman, B. 1973, ApJ, 183, 863 


\section{FIGURE LEGENDS}

Figure 1. A graph of the observed number of counts above the mean background level versus published $H \alpha$ fluxes (in units of $10^{-18} \mathrm{erg} \mathrm{cm}^{-2} \mathrm{~s}^{-1}$ ) from Hodge et al. (1989) for 15 bright (surface brightness $\mathrm{I} \geq 10^{-13} \mathrm{erg} \mathrm{cm}^{-2} \mathrm{sec}^{-1}$ ) isolated regions in the galaxy. The best fit slope through the data determines the calibration constant $\gamma=2.22 \times 10^{-18} \mathrm{erg} \mathrm{cm}^{-2} \mathrm{~s}^{-1}$ count $^{-1}$.

Figure 2. The $\sim 7.5^{\prime} \times 7.5^{\prime}$ continuum subtracted $H \alpha$ image of NGC 6822 illustrating the distribution of the bright, halo, diffuse and field $H \alpha$ emission regions. North is at the top and east is to the left. For reference, the giant H II regions Hubble I and III are to the top right. The contours, corresponding to surface brightnesses of $I=1.7 \times 10^{-15}, 4.6 \times 10^{-16}$ and $1.4 \times 10^{-16} \mathrm{erg} \mathrm{s}^{-1} \mathrm{~cm}^{-2} \operatorname{arcsec}^{-2}$, separate the bright, halo, diffuse and field regions.

Figure 3. The distribution of all OB stars $(\mathrm{V} \leq 21$ and $(\mathrm{B}-\mathrm{V}) \leq 0.5)$ in the inner kpc of NGC 6822 . Photometric coverage of the galaxy was restricted to inside the "T" shaped region outlined. The location of field OB stars are marked by plus signs while the OB stars in the bright, halo and diffuse regions are marked by a small square.

Figure 4. The luminosity functions for all blue $((\mathrm{B}-\mathrm{V}) \leq 0.5)$ stars in the survey as well those in the bright, halo, diffuse and field ionized gas environments. An arbitrary offset in the ordinate has been applied for the purposes of clarity. Overlaid on the data are the weighted least squares fits (to a limiting magnitude of $\mathrm{V}=19$ for the bright region and $\mathrm{V}=19.5$ elsewhere).

Figure 5. The $H \alpha$ image of the giant H II regions, Hubble I and III. Overlaid are the OB stars within the bright and halo emission regions

Figure 6. The continuum subtracted $H \alpha$ image of the central $8.7^{\prime} \times 8.7^{\prime}$ of M33 illustrating the distribution of the bright, halo, diffuse and field $H \alpha$ emission regions. North is at the top and east is to the left. For orientation the giant H II region NGC 595 is located in the top right-hand corner of the image and the center of the galaxy coincides roughly with the center of the image. 


\begin{tabular}{|c|c|c|c|c|c|c|c|c|c|c|}
\hline \multirow[t]{2}{*}{$\mathrm{V} \mathrm{MAG}^{*}$} & \multicolumn{2}{|c|}{$\overline{\text { BRIGHT }}$} & \multicolumn{2}{|c|}{ 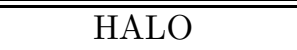 } & \multicolumn{2}{|c|}{ 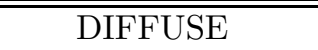 } & \multicolumn{2}{|c|}{$\overline{\overline{\text { FIELD }}}$} & \multicolumn{2}{|c|}{ 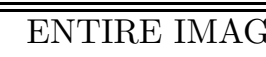 } \\
\hline & \# stars & $\%$ region & \# stars & $\%$ region & \# stars & $\%$ region & \# stars & $\%$ region & \# stars & $\%$ reg \\
\hline "V<18 & 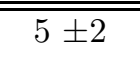 & 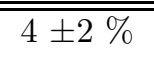 & 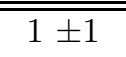 & $2 \pm 2 \%$ & 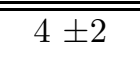 & $2 \pm 1 \%$ & 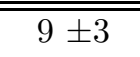 & 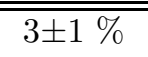 & 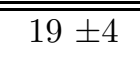 & 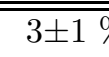 \\
\hline $18<\mathrm{V}<19$ & $16 \pm 4$ & $13 \pm 4 \%$ & $8 \pm 3$ & $10 \pm 5 \%$ & $6 \pm 2$ & $3 \pm 1 \%$ & $25 \pm 5$ & $7 \pm 2 \%$ & $55 \pm 7$ & $8 \pm$ \\
\hline $19<\mathrm{V}<20$ & $35 \pm 6$ & $30 \pm 8 \%$ & $27 \pm 5$ & $35 \pm 11 \%$ & $44 \pm 7$ & $25 \pm 6 \%$ & $88 \pm 9$ & $25 \pm 4 \%$ & $194 \pm 14$ & $27 \pm 3$ \\
\hline $20<\mathrm{V}<21$ & $62 \pm 8$ & $52 \pm 11 \%$ & $41 \pm 6$ & $53 \pm 14 \%$ & $121 \pm 11$ & $69 \pm 11 \%$ & $232 \pm 15$ & $67 \pm 8 \%$ & $456 \pm 21$ & $63 \pm$ \\
\hline $\mathrm{V} \leq 21$ (Total) & $118 \pm 11$ & & $77 \pm 8$ & & $175 \pm 13$ & & $354 \pm 19$ & & $724 \pm 27$ & \\
\hline
\end{tabular}

Table 1: The Distribution of Blue Stars in Four Ionized Gas Environments in N6822.

\footnotetext{
* Note : Only stars with $(\mathrm{B}-\mathrm{V}) \leq 0.5$
} 
${ }^{a}$ All stars with $(\mathrm{B}-\mathrm{V}) \leq 0.5$ and $\mathrm{V} \leq 21$.

${ }^{b}$ In units of $10^{-16} \mathrm{erg} \mathrm{s}^{-1} \mathrm{~cm}^{-2} \operatorname{arcsec}^{-2}$. This is measured above the average background.

${ }^{c}$ In units of $\mathrm{L}_{39} \equiv 10^{39} \mathrm{erg} \mathrm{s}^{-1}$.

${ }^{d}$ In units of $1.86 \times 10^{37} \mathrm{erg} \mathrm{s}^{-1} \mathrm{star}^{-1}$.

\begin{tabular}{lccccc}
\hline \hline & BRIGHT & HALO & DIFFUSE & FIELD & ENTIRE IMAGE \\
\hline \hline Total \# of Stars ${ }^{\text {a }}$ & 118 & 77 & 175 & 354 & 724 \\
Area Covered $\left(\mathrm{kpc}^{2}\right)$ & 0.06 & 0.13 & 0.56 & 1.04 & 1.79 \\
Surface Density of Stars $\left(\mathrm{kpc}^{-2}\right)$ & 1967 & 592 & 312 & 340 & 404 \\
Average Surface Brightness [I] b & 71.56 & 8.03 & 0.99 & 0.20 & 1.44 \\
Total Luminosity [L $\left._{o b}\right]^{\mathrm{c}}$ & 2.19 & 0.53 & 0.83 & 0.29 & 3.85 \\
Relative Luminosity per Star ${ }^{\mathrm{d}}$ & 1 & 0.37 & 0.26 & 0.04 & 0.28 \\
\hline \hline
\end{tabular}

Table 2: Average Properties of the Four Ionized Gas Environments. 
* Note: All stars with $\mathrm{V} \leq 21$ and all fluxes in units of $\mathrm{L}_{39} \equiv 10^{39} \mathrm{erg} \mathrm{s}^{-1}$.

${ }^{a}$ Minimum: no corrections applied.

${ }^{b}$ Incompleteness: corrected for incompleteness in stars brighter than $\mathrm{V}=21$.

${ }^{c}$ Best estimate: corrected for stars brighter and fainter than $\mathrm{V}=21$.

${ }^{d}$ Conservative estimate: corrected only for stars with $\mathrm{V}>21$.

${ }^{e}$ Observed fluxes are measured above the average sky background.

\begin{tabular}{|c|c|c|c|c|c|}
\hline & BRIGHT & HALO & $\begin{array}{c}\text { DIFFUSE } \\
(\mathrm{CASE} A / \mathrm{B})\end{array}$ & $\begin{array}{c}\text { FIELD } \\
\text { (CASE A/B) }\end{array}$ & $\begin{array}{c}\text { TOTAL } \\
(\mathrm{CASE} \mathrm{A/B})\end{array}$ \\
\hline Minimum Flux ${ }^{a}$ & 3.16 & 1.10 & $0.95 / 2.29$ & $1.72 / 4.16$ & $6.93 / 10.71$ \\
\hline Incompleteness $(\mathrm{V}<21)^{\mathrm{b}}$ & 4.28 & 1.52 & $1.36 / 3.28$ & $2.33 / 5.64$ & $9.75 / 14.72$ \\
\hline Best Estimate $^{c}$ & 4.32 & 1.55 & $1.39 / 3.33$ & $2.39 / 5.73$ & $9.65 / 14.93$ \\
\hline Conservative Estimate $^{d}$ & 3.19 & 1.22 & $0.96 / 2.32$ & $1.74 / 4.22$ & $7.11 / 10.95$ \\
\hline Observed Flux & 2.19 & 0.53 & 0.83 & 0.29 & 3.85 \\
\hline
\end{tabular}

Table 3: Observed and Predicted $\mathrm{H}_{\alpha}$ Flux in the Four Ionized Gas Environments. 
${ }^{a}$ Predicted $H \alpha$ luminosity accounting for incompleteness and/or differential reddening (as in Table 3). All stars with $\mathrm{V} \leq 21$ and all fluxes in units of $\mathrm{L}_{39}$.

${ }^{b}$ Observed luminosities have been background subtracted.

${ }^{c}$ In units of $1.86 \times 10^{37} \mathrm{erg} \mathrm{s}^{-1} \operatorname{arcsec}^{-2} \operatorname{star}^{-1}$.

\begin{tabular}{|c|c|c|c|}
\hline & BRIGHT & HALO & $\begin{array}{c}\text { COMBINED } \\
(\mathrm{BRIGHT}+\mathrm{HALO}) \\
\end{array}$ \\
\hline Minimum Flux ${ }^{\mathrm{a}}$ & 1.24 & 0.0035 & 1.24 \\
\hline Incompleteness $(\mathrm{V} \leq 21)^{\mathrm{a}}$ & 1.72 & 0.0080 & 1.73 \\
\hline Best Estimate $^{a}$ & 1.74 & 0.0082 & 1.73 \\
\hline Conservative Estimate $^{a}$ & 1.25 & 0.0049 & 1.26 \\
\hline Observed Luminosity $\left[\mathrm{L}_{o b}\right]^{\mathrm{b}}$ & 0.75 & 0.05 & 0.80 \\
\hline \# Stars Observed $\left[N_{o b}\right]$ & 40 & 3 & 43 \\
\hline \# Stars with $T_{e f f, L}<t_{e f f}<T_{e f f, U}{ }^{\mathrm{c}}$ & 35 & 3 & 38 \\
\hline Relative Luminosity per star $\left[\mathrm{L}_{o b} / N_{o b}\right]^{\mathrm{c}}$ & 1.01 & 0.89 & 1.00 \\
\hline
\end{tabular}

Table 4: The Observed and Predicted H $\alpha$ Fluxes and OB Star Content in Hubble I and III. 
${ }^{a}$ The brightest stars are selected such that $M_{V}<-7.45$ in both galaxies and $(\mathrm{B}-\mathrm{V})<0.4$ for M33 and $(\mathrm{B}-\mathrm{V}) \leq 0.5$ for NGC 6822 . Note that $M_{V} \leq-7.45$ corresponds to $\mathrm{V} \leq 18$ in $\mathrm{M} 33$ and $\mathrm{V} \leq 17.5$ in NGC 6822 .

${ }^{b}$ Total number of stars to a limiting absolute magnitude $M_{V} \leq-4.45$ (corresponding to $\mathrm{V} \leq 21$ in $\mathrm{M} 33$ and $\mathrm{V} \leq 20$ in NGC 6822) for both galaxies. The colour cutoff as is above.

\begin{tabular}{llccccc}
\hline \hline & GALAXY & $\begin{array}{c}\text { BRIGHT } \\
\text { \# of stars }\end{array}$ & $\begin{array}{c}\text { HALO } \\
\text { \# of stars }\end{array}$ & $\begin{array}{c}\text { DIFFUSE } \\
\text { \# of stars }\end{array}$ & $\begin{array}{c}\text { FIELD } \\
\text { \# of stars }\end{array}$ & $\begin{array}{c}\text { TOTAL } \\
\text { \# of stars }\end{array}$ \\
\hline Brightest Stars $^{\text {a }}$ & M33 & $22 \pm 5$ & $15 \pm 4$ & $7 \pm 3$ & $11 \pm 3.3$ & $55 \pm 7$ \\
& NGC 6822 & $2 \pm 1$ & 0 & $2 \pm 1$ & $6 \pm 2$ & $10 \pm 3$ \\
\multirow{2}{*}{ Total in Survey } & b & & & & & \\
& MG3 & $390 \pm 20$ & $688 \pm 26$ & $519 \pm 23$ & $583 \pm 24$ & $2180 \pm 47$ \\
& NGC 6822 & $84 \pm 9$ & $51 \pm 7$ & $119 \pm 11$ & $224 \pm 15$ & $481 \pm 22$ \\
\hline \hline
\end{tabular}

Table 5: The Distribution of OB Stars in M33 and NGC 6822. 
All predicted fluxes are expressed as a fraction of the observed $H \alpha$ flux in the particular ionized gas environment of the galaxy. Magnitude and colour cutoffs in respectively, M33 and NGC 6822 are, respectively, $\mathrm{V} \leq 21$ and $(\mathrm{B}-\mathrm{V}) \leq 0.4$ and $\mathrm{V} \leq 20.5$ and $(\mathrm{B}-\mathrm{V}) \leq 0.55$.

${ }^{a}$ Minimum flux M33: no corrections applied.

${ }^{b}$ Minimum flux NGC 6822: no corrections applied.

${ }^{c}$ Conservative estimate M33: corrected only for stars with $\mathrm{V}>21$.

${ }^{d}$ Conservative estimate NGC 6822: corrected only for stars with $\mathrm{V}>20.5$.

${ }^{e}$ Best estimate M33: corrected for stars with $\mathrm{V} \leq 21$ and $\mathrm{V}>21$.

${ }^{f}$ Best estimate NGC 6822: corrected for stars with $\mathrm{V} \leq 20.5$ and $\mathrm{V}>20.5$.

${ }^{g}$ Observed fluxes are in units of $\mathrm{L}_{39} \equiv 10^{39} \mathrm{erg} \mathrm{s}^{-1} \operatorname{arcsec}^{-2}$. 


\begin{tabular}{|c|c|c|c|c|c|c|}
\hline & GALAXY & $\begin{array}{l}\text { BRIGHT } \\
\text { (CASE B) }\end{array}$ & $\begin{array}{c}\text { HALO } \\
\text { (CASE B) }\end{array}$ & $\begin{array}{l}\text { DIFFUSE } \\
\text { (CASE A) }\end{array}$ & $\begin{array}{c}\text { FIELD } \\
\text { (CASE A) }\end{array}$ & TOTAL \\
\hline \multirow[t]{2}{*}{ MINIMUM } & M33 $^{\mathrm{a}}$ & 1.29 & 1.58 & 1.30 & 17.09 & 2.29 \\
\hline & NGC $6822^{\mathrm{b}}$ & 1.14 & 1.54 & 0.91 & 4.07 & 1.46 \\
\hline \multirow[t]{2}{*}{ CONSERVATIVE } & $\mathrm{M} 33^{\mathrm{c}}$ & 2.10 & 3.62 & 2.98 & 40.11 & 5.28 \\
\hline & NGC $6822^{\mathrm{d}}$ & 1.20 & 1.74 & 1.00 & 4.66 & 1.49 \\
\hline \multirow[t]{2}{*}{ BEST ESTIMATE } & M33 ${ }^{\mathrm{e}}$ & 3.97 & 8.35 & 6.20 & 88 & 6.51 \\
\hline & NGC $6822^{\mathrm{f}}$ & 1.29 & 1.73 & 1.13 & 4.93 & 1.59 \\
\hline \multirow[t]{2}{*}{ OBSERVED $\mathrm{H} \alpha^{\mathrm{g}}$} & M33 & 3.78 & 2.57 & 0.95 & 0.06 & 7.36 \\
\hline & NGC 6822 & 2.19 & 0.53 & 0.83 & 0.29 & 3.85 \\
\hline
\end{tabular}

Table 6: Predicted and Observed H $\alpha$ Fluxes in the Four Ionized Gas Environments of M33 and NGC 6822. 